01

\title{
Анализ добротности вынужденных колебаний дробного линейного осциллятора
}

\author{
(C) Р.И. Паровик
}

Институт космофизических исследований и распространения радиоволн ДВО РАН, 684034 Камчатский край, с. Паратунка, Россия

Камчатский государственный университет им. Витуса Беринга,

683032 Петропавловск-Камчатский, Россия

e-mail: romano84@mail.ru

Поступило в Редакцию 6 июня 2019 г.

В окончательной редакции 2 декабря 2019г.

Принято к публикации 27 января 2020 г.

С помощью метода гармонического баланса получены аналитические формулы для расчета амплитудночастотной и фазово-частотной характеристик, а также добротности вынужденных колебаний дробного линейного осциллятора. Установлено, что исследуемые характеристики зависят от диссипативных свойств среды - эффектов памяти, которые описываются производными дробных порядков. Показано, что дробные порядки влияют на характер затухания колебательного процесса и связаны с его добротностью. Расчетные кривые характеристик вынужденных колебаний дробного линейного осциллятора показали, что дробные порядки можно рассматривать как управляющие параметры колебательного процесса в диссипативной среде.

Ключевые слова: добротность, амплитудно-частотная характеристика, фазово-частотная характеристика, дробные производные, память.

DOI: $10.21883 / J T F .2020 .07 .49436 .233-19$

\section{Введение}

Дробные осцилляторы характеризуют колебательные процессы в диссипативных средах и рассматриваются в рамках дробной динамики [1]. С математической точки зрения дробные осцилляторы можно описать с помощью интегродифференциальных уравнений с разностными ядрами - степенными функциями памяти, которые характеризуют наследственные свойства среды, или с помощью производных дробных порядков [2]. Введение дробных производных в модельное уравнение позволяет учесть динамическую память (гистерезис), которая возникает в наследственной механике в результате диссипации энергии деформации при наличии вязкоупругости [3], а также в магнитном поле [4] или в электронике [5].

Особый интерес представляет анализ вынужденных колебаний дробных осцилляторов с целью установления связи между их характеристиками и свойствами среды - порядками дробных производных.

В работе [6] были исследованы вынужденные колебания дробного линейного осциллятора. Была выведена аналитическая формула, которая связывает порядок дробной производной в инерциальном члене с добротностью $Q$. Показано, что при больших значениях добротности результаты расчета в рамках классической и дробной моделей осциллятора практически неразличимы.

Продолжением работы [6] является настоящая работа, в которой мы исследовали дробный линейный осциллятор с учетом вязкого трения и внешнего гармонического воздействия, с помощью метода гармонического баланса получили аналитические формулы для расчета амплитудно-частотной и фазово-частотной характеристик (АЧХ и ФЧХ), а также добротности, и показали, что дробные порядки производных можно рассматривать как управляющие параметры колебательного процесса в диссипативной среде.

\section{Модель дробного линейного осциллятора}

Рассмотрим следующее модельное интегродифференциальное уравнение точечного осциллятора:

$$
\begin{aligned}
& m_{\beta} \int_{0}^{t} K_{1}(t-\tau) \ddot{x}(\tau) d \tau+\lambda \int_{0}^{t} K_{2}(t-\tau) \dot{x}(\tau) d \tau \\
& \quad+\sigma_{0}^{\beta} x(t)=F \cos (\omega t),
\end{aligned}
$$

где $K_{1}(t-\tau)$ и $K_{2}(t-\tau)$ - разностные ядра, характеризующие память (свойство среды), $\lambda-$ коэффициент трения, $x(t)$ - функция смещения, $\sigma_{0}^{\beta}-$ частота собственных колебаний, $f$ и $\omega-$ амплитуда и частота внешних гармонических колебаний, $m_{\beta}$ - масса точечного осциллятора, $1<\beta<2-$ параметр, характеризующий степень влияния памяти в колебательной среде.

Уравнение (1) описывает точечный линейный осциллятор, который учитывает эффекты памяти как в инерциальной составляющей, так и в составляющей, отвечающей за демпфирование.

Необходимо отметить, что функции памяти могут быть произвольными и выбираются, исходя из свойств среды. Известно, что существуют два предельных случая: когда функции памяти являются $\delta$-функциями и 
когда они являются функциями Хевисайда. В первом случае можно говорить об отсутствии памяти в динамической системе, а во втором случае - о полном ее наличии [7].

Мы же будем рассматривать промежуточный случай, когда функции памяти имеют степенной вид. В этой ситуации динамическая система постепенно „теряет память“ о первоначальном воздействии силы, что приводит к диссипации энергии. Степенные распределения очень часто встречаются в различных областях знаний [8].

Пусть функции памяти в уравнении (1) имеют вид

$$
\begin{array}{cl}
K_{1}(t-\tau)=\frac{(t-\tau)^{1-\beta}}{\Gamma(2-\beta)}, & K_{2}(t-\tau)=\frac{(t-\tau)^{-\gamma}}{\Gamma(1-\gamma)}, \\
1<\beta<2, & 0<\gamma<1 .
\end{array}
$$

Скейлинговые параметры $\beta$ и $\gamma$ в соотношениях (2) характеризуют интенсивность диссипации энергии в колебательной среде. Если подставить представления (2) в модельное уравнение (1) и, учитывая определения производных дробных порядков в смысле Герасимова-Капуто $[9,10]$

$$
\begin{aligned}
\partial_{0 t}^{\beta} x(\tau) & =\frac{1}{\Gamma(2-\beta)} \int_{0}^{t} \frac{\ddot{x}(\tau) d \tau}{(t-\tau)^{\beta-1}}, \\
\partial_{0 t}^{\gamma} x(\tau) & =\frac{1}{\Gamma(1-\gamma)} \int_{0}^{t} \frac{\dot{x}(\tau) d \tau}{(t-\tau)^{\gamma}},
\end{aligned}
$$

приходим к следующему уравнению:

$$
\partial_{0 t}^{\beta} x(\tau)+\lambda \partial_{0 t}^{\gamma} x(\tau)+\omega_{0}^{\beta} x(t)=f \cos (\omega t),
$$

где $\lambda=\Lambda / m_{\beta}, f=F / m_{\beta}, \omega_{0}^{\beta}=\sigma_{0}^{\beta} / m_{\beta}$.

Уравнение (4) описывает наследственный процесс вынужденных колебаний с учетом трения. В предельном случае, когда $\beta=2, \gamma=1 \mathrm{y}$, уравнение (4) переходит в классический гармонический осциллятор с трением и внешним гармоническим воздействием.

Для уравнения (4) в работе автора [11] были исследована задача Коши, получено ее аналитическое решение в терминах обобщенной функции Райта, построены и исследованы осциллограммы и фазовые траектории при различных значениях $\beta$ и $\gamma$. В работе [12] исследованы точки покоя.

\section{Характеристики дробного линейного осциллятора}

В дальнейшем объектом нашего исследования будут установившиеся колебания дробного осциллятора (4). С помощью метода гармонического баланса мы выведем аналитические формулы для расчета амплитуды и фазы установившихся колебаний, а также добротности дробного осциллятора (4). Решение уравнения (4), согласно методу гармонического баланса, ищем в виде [13]

$$
x(t)=A \cos (\omega t+\delta)=A \cos (u) .
$$

С учетом (3) уравнение (4) перепишем в виде

$$
\begin{gathered}
\frac{1}{\Gamma(2-\beta)} \int_{0}^{t} \frac{\ddot{x}(\tau) d \tau}{(t-\tau)^{\beta-1}}+\frac{\lambda}{\Gamma(1-\gamma)} \int_{0}^{t} \frac{\dot{x}(\tau) d \tau}{(t-\tau)^{\gamma}} \\
+\omega_{0}^{\beta} x(t)=f \cos (\omega t) .
\end{gathered}
$$

Тогда первое слагаемое в (4) с учетом представления (3) можно представить в виде

$$
\begin{aligned}
& \frac{1}{\Gamma(2-\beta)} \int_{0}^{t} \frac{\ddot{x}(\tau) d \tau}{(t-\tau)^{\beta-1}}=\frac{1}{\Gamma(2-\beta)} \int_{0}^{t} v^{1-\beta} \ddot{x}(t-v) d v \\
& =-\frac{A \omega^{2}}{\Gamma(2-\beta)} \int_{0}^{t} v^{1-\beta} \cos (\omega(t-v)+\delta) d v=-\frac{A \omega^{2}}{\Gamma(2-\beta)} \\
& \times \int_{0}^{t} v^{1-\beta}[\cos (\omega(t-v)) \cos (\delta)-\sin (\omega(t-v)) \sin (\delta)] d v \\
& =-\frac{A \omega^{2} \cos (\delta)}{\Gamma(2-\beta)} \int_{0}^{t} v^{1-\beta} \cos (\omega(t-v)) d v \\
& +\frac{A \omega^{2} \sin (\delta)}{\Gamma(2-\beta)} \int_{0}^{t} v^{1-\beta} \sin (\omega(t-v)) d v \\
& =-\frac{A \omega^{2} \cos (\delta)}{\Gamma(2-\beta)} \int_{0}^{t} v^{1-\beta}[\cos (\omega t) \cos (\omega \nu) \\
& +\sin (\omega t) \sin (\omega v)] d v+\frac{A \omega^{2} \sin (\delta)}{\Gamma(2-\beta)} \\
& \times \int_{0}^{t} v^{1-\beta}[\sin (\omega t) \cos (\omega t)-\cos (\omega t) \sin (\omega t)] d v \\
& =-\frac{A \omega^{2} \cos (\delta) \cos (\omega t)}{\Gamma(2-\beta)} \int_{0}^{t} v^{1-\beta} \cos (\omega \nu) d v \\
& -\frac{A \omega^{2} \cos (\delta) \sin (\omega t)}{\Gamma(2-\beta)} \int_{0}^{t} v^{1-\beta} \sin (\omega v) d v \\
& +\frac{A \omega^{2} \sin (\delta) \sin (\omega t)}{\Gamma(2-\beta)} \int_{0}^{t} v^{1-\beta} \cos (\omega v) d v \\
& -\frac{A \omega^{2} \sin (\delta) \cos (\omega t)}{\Gamma(2-\beta)} \int_{0}^{t} v^{1-\beta} \sin (\omega v) d v \\
& =[\sin (\delta) \sin (\omega t)-\cos (\delta) \cos (\omega t)] \frac{A \omega^{2}}{\Gamma(2-\beta)} \\
& \times \int_{0}^{t} v^{1-\beta} \cos (\omega v) d v-[\sin (\delta) \cos (\omega t)+\cos (\delta) \sin (\omega t)] \\
& \times \frac{A \omega^{2}}{\Gamma(2-\beta)} \int_{0}^{1} v^{1-\beta} \sin (\omega v) d v=\frac{-A \omega^{2} \cos (u)}{\Gamma(2-\beta)} \\
& \times \int_{0}^{1} v^{1-\beta} \cos (\omega v) d v-\frac{A \omega^{2} \sin (u)}{\Gamma(2-\beta)} \int_{0}^{t} v^{1-\beta} \sin (\omega v) d v .
\end{aligned}
$$


В случае установившихся колебаний при $t \rightarrow \infty$ интегралы в (7) можно записать так [14]:

$$
\begin{aligned}
& \int_{0}^{1} v^{1-\beta} \cos (\omega \nu) d v \approx \frac{\Gamma(2-\beta)}{\omega^{2-\beta}} \sin ((\beta-1) \pi / 2) \\
& =-\frac{\Gamma(2-\beta)}{\omega^{2-\beta}} \cos (\beta \pi / 2), \\
& \int_{0}^{1} v^{1-\beta} \sin (\omega v) d v \approx \frac{\Gamma(2-\beta)}{\omega^{2-\beta}} \cos ((\beta-1) \pi / 2) \\
& =-\frac{\Gamma(2-\beta)}{\omega^{2-\beta}} \sin (\beta \pi / 2) .
\end{aligned}
$$

С учетом этих соотношений получим

$$
\begin{aligned}
& \frac{1}{\Gamma(2-\beta)} \int_{0}^{t} \frac{\ddot{x}(\tau) d \tau}{(t-\tau)^{\beta-1}} \\
& \approx A \omega^{\beta}(\cos (u) \cos (\beta \pi / 2)-\sin (u) \sin (\beta \pi / 2)) .
\end{aligned}
$$

Аналогично второе слагаемое в (4) будет иметь вид

$$
\begin{aligned}
& \frac{\lambda}{\Gamma(1-\gamma)} \int_{0}^{1} \frac{\dot{x}(\tau) d \tau}{(t-\tau)^{\gamma}} \approx \lambda a \omega^{\gamma} \\
& \times(\cos (\gamma \pi / 2) \cos (u)-\sin (\gamma \pi / 2) \sin (u)) .
\end{aligned}
$$

С учетом (8), (9) и (5) уравнение (6) перепишем в виде

$$
\begin{aligned}
& A \omega^{\beta}(\cos (u) \cos (\beta \pi / 2)-\sin (u) \sin (\beta \pi / 2)) \\
& +\lambda A \omega^{\gamma}(\cos (\gamma \pi / 2) \cos (u)-\sin (\gamma \pi / 2) \sin (u)) \\
& +\omega_{0}^{A} A \cos (u)=f \cos (\omega t) .
\end{aligned}
$$

Примем во внимание, что $\dot{x}(t)=-A \omega \sin (u)$, $\ddot{x}(t)=-A \omega^{2} \cos (u)$ поэтому уравнение (10) можно представить в следующей форме:

$$
m \ddot{x}(t)+p \dot{x}(t)+s^{2} x(t)=f \cos (\omega t),
$$

где

$$
\begin{gathered}
m=-\omega^{\beta-2} \cos (\beta \pi / 2), \\
p=\omega^{\beta-1} \sin (\beta \pi / 2)+\lambda \omega^{\gamma-1} \sin (\gamma \pi / 2), \\
s^{2}=\omega_{0}^{\beta}+\lambda \omega^{\gamma} \cos (\gamma \pi / 2) .
\end{gathered}
$$

Отметим, что уравнение (11) представляет собой классический линейный осциллятор с трением и внешним гармоническим воздействием, для которого известны соотношения для АЧХ и ФЧХ характеристик:

$$
A=\frac{f}{\sqrt{U^{2}+W^{2}}}, \quad \delta=\arctan \left(-\frac{W}{U}\right),
$$

где $U=s^{2}-\omega^{2} m, W=\omega p$.
Формулы (12) для АЧХ и ФЧХ можно переписать следующим образом:

$A=\frac{f}{\sqrt{\omega^{2 \beta}+\omega_{0}^{2 \beta}+\lambda \omega^{\gamma}\left(\lambda \omega^{\gamma}+2 \omega^{\gamma} \cos ((\beta-\gamma) \pi / 2)+2 \omega_{0}^{\beta} \cos (\gamma \pi / 2)\right)+2 \omega_{0}^{\beta} \omega^{\beta} \cos (\beta \pi / 2)}}$,

$$
\delta=\arctan \left(-\frac{\left(\omega^{\beta} \sin (\beta \pi / 2)+\lambda \omega^{\gamma} \sin (\gamma \pi / 2)\right)}{\omega_{0}^{\beta}+\lambda \omega^{\gamma} \cos (\gamma \pi / 2)+\omega^{\beta} \cos (\beta \pi / 2)}\right) .
$$

Отметим, что в частном случае в $(13)$, когда $\beta=2 \gamma$, $\lambda=2 b^{\gamma}$, мы приходим к формулам (14) и (15) из работы $[15]$ с учетом $\phi=0$. Также заметим, что при $\lambda=0$ в (13) мы приходим к формулам (4) и (7), полученным в работе [6].

Добротность $Q$ дробного линейного осциллятора (4) можно определить, согласно уравнению (9), из соотношения

$$
Q=\frac{s}{p}=\frac{\sqrt{\omega_{0} \beta}+\lambda \omega^{\gamma} \cos (\gamma \pi / 2)}{\omega^{\beta-1} \sin (\beta \pi / 2)+\lambda \omega^{\gamma-1} \sin (\gamma \pi / 2)} .
$$

Из формулы (14) следует, что добротность $Q$ зависит от дробных параметров $\beta$ и $\gamma$.

В работе [6] была выведена формула при отсутствии трения $(\lambda=0)$, которая связывала дробный порядок производной в инерциальной составляющей колебательного процесса и его добротность. Далее было показано, что при уменьшении значений дробного показателя, добротность $Q$ уменьшается и, следовательно, колебательный процесс быстрее затухает. Подтвердим с помощью формул (13) и (14) ранее полученные результаты и покажем, что при уменьшении значений дробного порядка производной в диссипативном члене происходит увеличении добротности $Q$.

\section{Результаты расчетов характеристик}

В аналитических формулах (13) и (14) для простоты мы положим: $\lambda=0.15, f=0.5, \omega_{0}^{\beta}=m_{\beta}=1$. Результаты расчетов приведены на рис. $1-3$.

На рис. 1 при фиксированном значении $\gamma=1$ и различных значениях параметра $\beta$ построены расчетные кривые АЧХ и ФЧХ дробного линейного осциллятоpa (4).

Кривые АЧХ или резонансные кривые характеризуются резонансной частотой $\omega_{R}$ и соответствующей ей максимальной амплитудой $A_{R}$, которые можно найти из решения уравнения $d A / d \omega=0$ с учетом формулы (13). Из рис. $1, a$ а мы получим для кривой $1-\omega_{r}=0.994$, $A_{R}=6.685$, для кривой $2-\omega_{R}=0.936, A_{R}=2.177$, для кривой $3-\omega_{R}=0.7969, A_{R}=1.218$. Из полученных значений $\omega_{R}$ следует, что резонансная частота смещается в область низших частот, а максимальная амплитуда, которая ей соответствует, уменьшается. Поэтому добротность $Q$ определяет ширину резонансной кривой. И, следовательно, как видно на рис. 1, $a$, при 


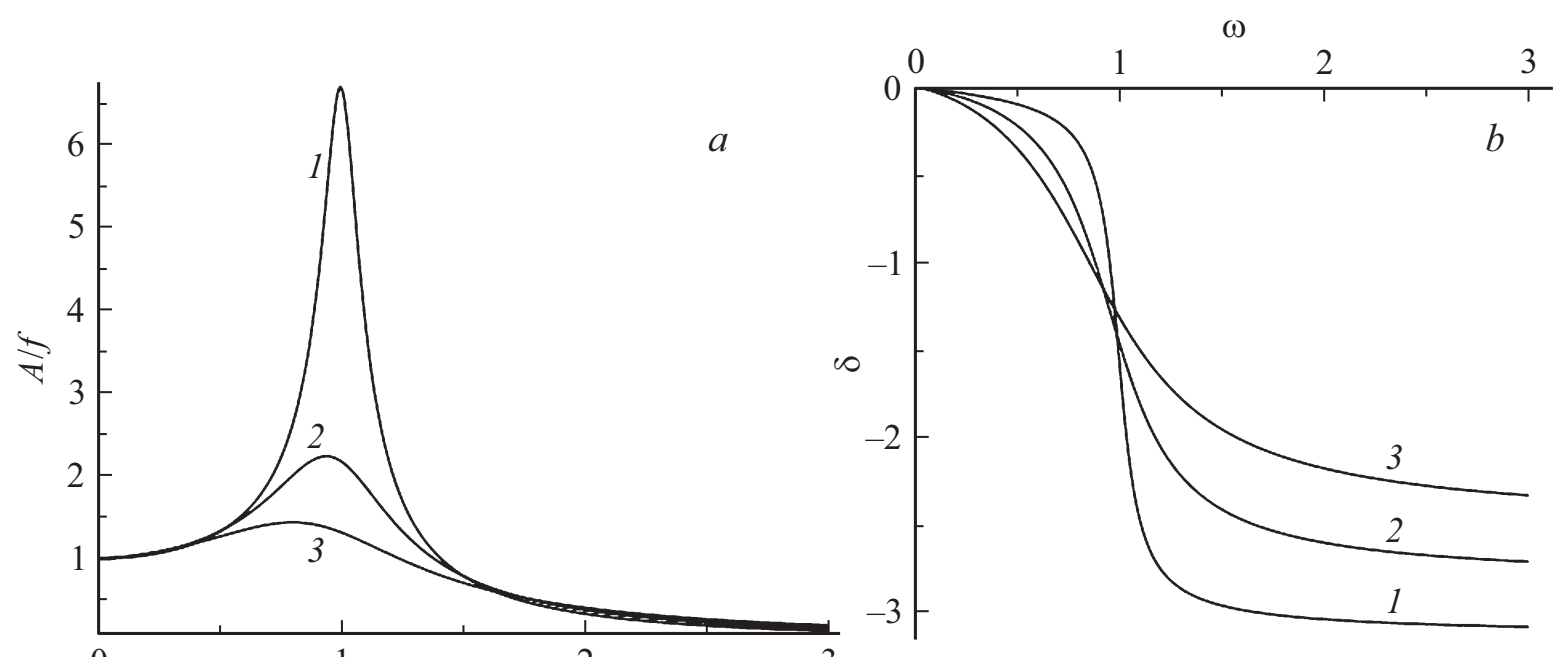

$\omega$

Рис. 1. АЧХ $(a)$ и ФЧХ $(b)$, полученные по формулам (13) при $\gamma=1$, в зависимости от значений параметра $\beta$ : кривая $1-2$; кривая $2-1.8$; кривая $3-1.6$.
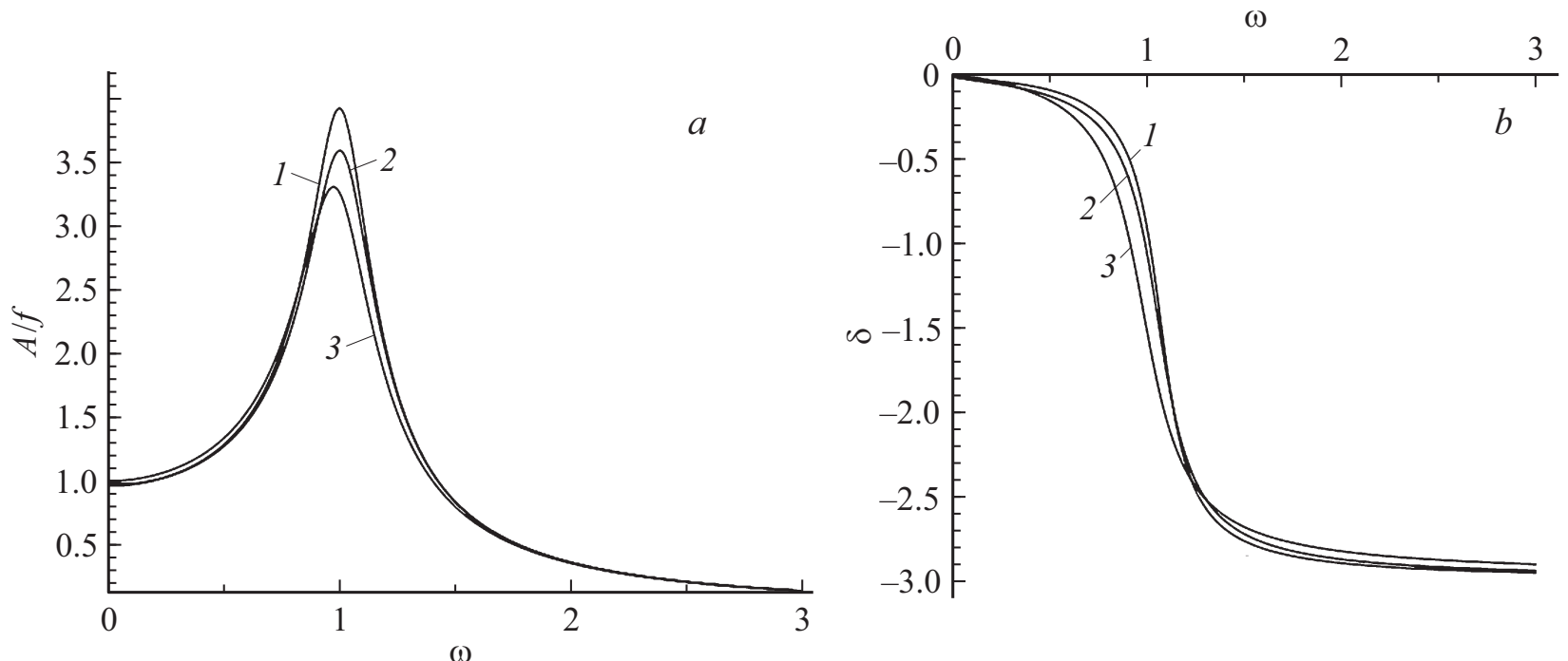

Рис. 2. АЧХ $(a)$ и ФЧХ $(b)$, полученные по формулам (13) при $\beta=1.9$, в зависимости от значений параметра $\gamma$ : кривая $1-1$; кривая $2-0.4$; кривая $3-0.2$.

уменьшении значений параметра $\beta \rightarrow 1$, добротность $Q$ уменьшается, и колебания быстрее затухают.

На рис. $1, b$ приведен фазовый сдвиг - запаздывание смещения $x(t)$ по фазе от внешней силы в зависимости от различных значений $\beta$. В классическом случае $(\beta=2)$ предельная величина фазового сдвига при $\omega \rightarrow \infty$ составляет $-\pi$. При уменьшении значений $\beta \rightarrow 1$ величина фазового сдвига уменьшается. Необходимо отметить, что расчетные кривые АЧХ и ФЧХ перегруппировываются со временем в обратный порядок, что характерно для эффекта памяти.

На рис. 2 приведены расчетные кривые АЧХ и ФЧХ при фиксированном значении $\beta=1.9$ и различных значениях параметра $\gamma$.
На рис. 2, а для резонансных кривых были рассчитаны по формуле (13) параметры $\omega_{R}$ и $A_{R}$ : для кривой $1-\omega_{R}=0.974$, для кривой $2-\omega_{R}=1.001$, $A_{R}=3.587$, для кривой $3-\omega_{R}=0.999, A_{R}=3.918$. Мы видим, что резонансная частота $\omega_{r}$ принимает значения, близкие к единице, а максимальная амплитуда $A_{R}$ увеличивается. Поэтому мы можем сделать вывод о том, что при $\gamma \rightarrow 0$ добротность $Q$ увеличивается.

При фазовый сдвиг имеет примерно одинаковую величину (рис. $2, b)$.

На рис. 3 приведена поверхность, полученная по формуле (14) для добротности $Q(\beta, \gamma)$. Мы видим, что добротность будет увеличиваться при уменышении 


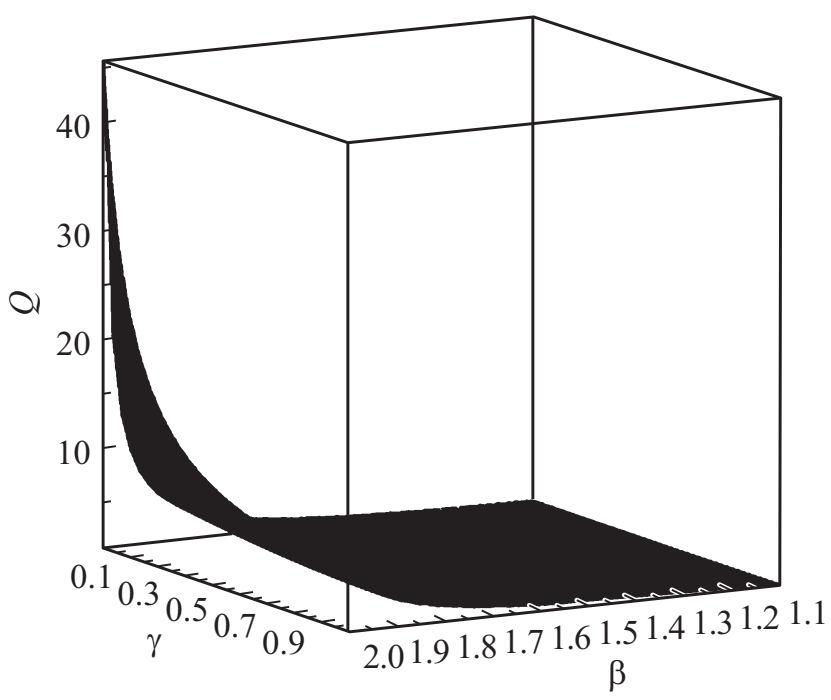

Рис. 3. Поверхность, характеризующая добротность $Q$ дробного осциллятора (4), в зависимости от значений параметров $\beta$ и $\gamma$, полученная по формуле (14).

параметра $\gamma$ и уменьшаться при уменьшении параметра $\beta$, что подтверждает анализ расчетных кривых АЧХ и ФЧХ на рис. 1 и 2.

\section{Заключение}

В работе получены методом гармонического баланса аналитические формулы для расчета АЧХ, ФЧХ и добротности $Q$ дробного линейного осциллятора (4). Эти характеристики колебательного процесса с памятью зависят от порядков $\beta$ и $\gamma$ дробных производных, которые входят в модельное уравнение (4). Анализ характеристик показал, что при уменьшении параметра $\beta \rightarrow 1$ происходит уменьшение добротности $Q$, а при уменьшении параметра $\gamma \rightarrow 0$, наоборот, происходит увеличение добротности $Q$. Параметры $\beta$ и $\gamma$ характеризируют интенсивность диссипации энергии колебательной системы. Поэтому, если мы рассматриваем колебательный процесс, в котором хотим учесть трение, то это можно сделать не только с помощью дополнительного слагаемого, отвечающего за демпфирование, но и с помощью дробной силы инерции с параметром $\beta$.

\section{Финансирование работы}

Работа выполнена при финансовой поддержке гранта президента РФ № МК-1152.2018.1.

\section{Конфликт интересов}

Автор заявляет, что у него нет конфликта интересов.

\section{Список литературы}

[1] Petras I. Fractional-Order Nonlinear Systems. Modeling, Analysis and Simulation. Beijing and Springer-Verlag, Berlin Heidelberg: Springer, 2011. $218 \mathrm{p}$.

[2] Parovik R.I. // Вестник Южно-Уральского гос. ун-та. Cер.: Математическое моделирование и программирование. 2018. Т. 11. № 2. С. 108-122.

[3] Caputo M., Carcione J.M. // Rheol Acta. 2011. N 50. P. 107-115. DOI: 10.1007/s00397-010-0524-z

[4] Mankin R., Laas K., Laas T., Paekivi S. / Phys. Rev. E. 2018. Vol. 97. P. 012145. DOI: 10.1103/PhysRevE.97.012145

[5] Kumar S., Gupta M., Kumar D. // Intern. J. Electron. 2019. Vol. 106. N 4. P. 581-598. DOI: $10.1080 / 00207217.2018 .1545260$

[6] Pskhu A.V., Rekhviashvili S.S. // Tech. Phys. Lett. 2018. Vol. 44. N 12. P. 1218-1221. DOI: $10.1134 / \mathrm{S} 1063785019010164$

[7] Бутенков C.A. // Известия Южного федерального ун-та. Техснические науки. 2011. Т. 121. № 8. С. 199-209.

[8] Schroeder M. Fractals, Chaos, Power Laws: Minutes from an Infinite Paradise. NY.: W.H. Freeman, 1991.

[9] Герасимов А.Н. // АН СССР. Прикладная математика и механика. 1948. Т. 12. С. 529-539.

[10] Caputo M. Elasticit'a e dissipazione. Bologna: Zanichelli, 1969. $150 \mathrm{p}$.

[11] Паровик Р.И. // Компьютерные исследования и моделирование. 2015. Т. 7. № 5. С. 1001-1022.

[12] Parovik R.I. Research of the stability of some hereditary dynamic system. IOP Conf. Series: J. Physics: Conf. Series. 2018. Vol. 1141. P. 012179. DOI: $10.1088 / 1742-6596 / 1141 / 1 / 012179$

[13] Li S., Niu J., Li X. // Chin. Phys. B. 2018. Vol. 27. N 12. P. 120502. DOI: $10.1088 / 1674-1056 / 27 / 12 / 120502$

[14] Kilbas A.A. Theory and Applications of Fractional Differential Equations / A.A. Kilbas, H.M. Srivastava, J.J. Trujillo. Amsterdam: Elsevier, 2006.

[15] Olivar-Romero F., Rosas-Ortiz O. Fractional Driven Damped Oscillator. IOP Conf. Series: J. Physics: Conf. Series. 2017. N 839. P. 012010. DOI: $10.1088 / 1742-6596 / 839 / 1 / 012010$ 